\title{
Promoting research utilisation in nursing: the role of the individual, organisation, and environment
}

Evidence-based nursing integrates the best evidence from research with clinical expertise, patient preferences, and existing resources into decision making about the health care of individual patients. ${ }^{1}$ To practise evidence-based nursing, clinical nurses need effective strategies for extracting relevant information from the many publications that are currently available. The quality of information that nurses demand and how effectively they evaluate and use it for clinical decision making will influence patient outcomes and, ultimately, the part nurses play in the delivery of health care.

The term "information society" was introduced in the 1980s to describe the information explosion precipitated by new technologies. ${ }^{2}$ Nursing practice is information intensive. Even 10 years ago, Mowry and Korpman estimated that nurses spent $40 \%$ of their time on information related tasks. ${ }^{3}$ The rapid growth of nursing information means that nurses cannot rely on knowledge acquired as students and must constantly update their practice.

Computerisation is simultaneously a major cause of the information explosion and a means of managing it. Already, computer systems enable healthcare professionals to access patient databases, research databases, clinical guidelines, and care protocols. The internet has emerged as a formidable medium for information provision and exchange. The next challenge for the software industry is to devise an effective means of organising the available information so that discriminating users can access it effectively.

In the 1980s, when research was finally recognised as an integral part of professional nursing, concern developed about the gap between research and practice. Researchers began to investigate barriers to research utilisation, using a variety of individual and organisational variables to study diffusion of information throughout organisations. ${ }^{4}$ They also surveyed nurses about their attitudes, resources, and experiences related to research utilisation, ${ }^{6}$ and experimented with different approaches to improving research utilisation. ${ }^{78}$

\section{How do nurses currently use information?}

Studies of nurses' use of printed materials show differences in awareness of research and availability of information resources, both of which are often limited in small hospitals and primary care settings. ${ }^{9}{ }^{10}$ One study found that, compared with small hospitals ( $\leqslant 500$ beds), hospitals with $>500$ beds were more likely to have nursing research coordinators, access to nursing research experts and nursing faculty, nursing research committees, libraries with nursing research journals, and to implement research utilisation programmes for their staff nurses. ${ }^{9}$ Other surveys suggest that most nurses read clinical or technical journals rather than research journals ${ }^{11}$ and rarely visit libraries. ${ }^{12}$ Nurses engaged in education or research, however, use these resources as well as indexes and computerised databases such as CINAHL and Medline. ${ }^{13}$

Patient specific data and reference sources are increasingly available in computerised hospital informatics systems. Nurses are willing to use these resources for clinical decision making and patient teaching when the information is relevant and the system is user friendly. In one study, ${ }^{13}$ nurses used online information to:

- Answer questions related to nursing diagnoses

- Prepare care plans

- Learn about drugs and disease processes

- Obtain information for patients

- Validate knowledge

- Investigate new interests

- Promote professional development.

Drug databases and nursing texts were used most frequently. Novice users accessed online information during night shifts or quiet times. More expert users accessed information on all shifts, but used patient specific, quick references during the day. Little is known about nurses' use of the internet, including whether they use it to answer clinical questions at home, in practice, or both. At 1 site, staff accessed internet nursing sites during quiet periods on the night shift.

\section{Unharnessing the power of information for practice}

Nurses are generally positive about evidence-based practice, but we know little about what determines their use of research findings. We do know that individual research utilisation is facilitated by organisational commitment to using research evidence. A systematic review of the literature on research utilisation, ${ }^{14}$ found that organisational factors explained $80-90 \%$ of the variance in research utilisation, environmental factors accounted for $5-10 \%$ of the variance, and individual characteristics contributed only 1-3\%. Multifaceted interventions directed at the individual, the organisation, and the environment are most likely to lead to changes in practice. ${ }^{15}$

Numerous researchers have investigated the causes of the research-practice gap and proposed solutions. Based on a literature review, Funk et al identified barriers to research utilisation which included the individual nurse, the organisation, the quality of the research, and the way that research results are communicated. ${ }^{16}$ Surveys using Funk's Barrier Scale showed a widespread recognition of these barriers. ${ }^{17}$ Reports on programmes aimed at promoting research utilisation suggest possible strategies to overcome them. ${ }^{7}$

\section{The individual nurse}

NETWORKING

Nurses who participate in research related projects promote research utilisation and are more likely than other nurses to apply research in practice. ${ }^{18}$ Nurses who understand the importance of evidence-based nursing might act as mentors to colleagues who are unaware of its potential impact on practice or the range of information available. Once persuaded, nurses can collaborate to promote research utilisation in the workplace. Peer mentoring and coaching are effective strategies for assisting colleagues to search for, evaluate, and apply information. ${ }^{13}{ }^{19}$ Nurses with access to the internet can pass on information, and groups of nurses can form journal clubs to share reading on topics of clinical interest. 


\section{PROMOTING ACCESSIBILITY}

Because the accessibility and quality of information have implications for the efficacy of clinical decision making, it is important that workplace reference materials are current and user friendly and that protocols and guidelines are based on the best research evidence. Evaluating and updating personal collections is also important.

\section{MANAGING TIME}

Prioritisation is important when time is limited. Strategic choices may include reading a research article or critical review instead of a clinical journal, or taking a course on how to understand or apply research findings. A trip to a health sciences or medical library to learn how to use CINAHL, Medline, or the Cochrane Library will make it easier to find useful articles. Pooling skills and resources can also increase research utilisation.

\section{The organisation}

Organisational characteristics, including size, location, and centralisation of decision making, have been associated with research utilisation, but conclusions about the importance of specific variables are inconsistent, ${ }^{14}$ perhaps because their effects vary in different settings. Programmes intended to improve research utilisation have been most successful when administrative support was available. ${ }^{1319}$ Evidence-based practice has been realised most completely in institutions that have adopted it as policy and have integrated it at all organisational levels. ${ }^{20}{ }^{21}$ At the University of Iowa Hospitals, an infrastructure supporting research use encompassed both high level management and frontline nurses. Duties relating to research utilisation were included in job descriptions; evidence-based practice was linked to quality assurance; and appropriate education was provided. Staff who used research to solve clinical problems were recognised and rewarded. The Iowa model used triggers to alert nurses to clinical problems in their workplaces and specified processes for solving them. Clinical nurses were allocated time and resources to be involved in all aspects of research utilisation including problem identification, assessment of evidence, change planning, implementation, and evaluation of change. Because the organisation placed a high priority on research utilisation, nurses were motivated to become involved. ${ }^{21}$

\section{The environment}

An information friendly environment links the clinical nurse to a variety of information brokers, the healthcare literature, and other evidence-based resources. The internet provides potential global connectedness for all nurses.

The development of tools for evidence-based nursing requires time and resources and is dependent on collaboration among healthcare organisations and academic institutions. As communication throughout the global healthcare community improves, pooling expertise on national and international levels becomes increasingly feasible. Centres for evidence-based nursing have been established in a number of countries and plans are underway to create an international centre with links to centres worldwide. These centres will provide access to critically appraised materials for nurses and organisations. Health science libraries are important resources for clinicians. Besides keeping up with new information resources, the librarian's role includes helping users to master information handling skills, and using technology to organise, synthesise, and filter information for scholarly, clinical, and institutional decision making.

\section{Conclusions}

In the future, information systems will integrate clinical data about patient treatments and preferences, available resources, expert opinion, and research evidence to provide nurses with current, patient specific information to address complex clinical issues. Nurses will be able to plan treatment with knowledgeable patients who will also have access to health related resources. Improved organisational and environmental support will be critical for nurses to work with increasingly sophisticated information systems.

JOAN ROYLE, RN, MScN JENNIFER BLYTHE, PhD, MLS

School of Nursing,

Faculty of Health Sciences,

McMaster University,

Hamilton, Ontario, Canada

1 DiCenso A, Cullum N, Ciliska D. Implementing evidence-based nursing: some misconceptions [implementation forum]. Evidence-Based Nursing 1998 Apr;1:38-40.

2 Naisbitt J. Megatrends: ten new directions for transforming our lives. New York: Warner Books, 1984.

Mowry MM, Korpman RA. Evaluating automated information systems. Nurs Econ 1987;5:7-12.

4 Brett JL. Organizational integrative mechanisms and adoption of innovations by nurses. Nurs Res 1989;38:105-10.

5 Champion VL, Leach A. Variables related to research utilization in nursing: an empirical investigation. J Adv Nurs 1989;14:705-10.

6 Funk SG, Champagne MT, Weise RA, et al. Barriers to using research findFunk SG, Champagne MT, Weise RA, et al. Barriers to using research fin
ings in practice: the clinician's perspective. Appl Nurs Res 1991;4:90-5.

ings in practice: the clinician's perspective. Appl Nurs Res 1991;4:90-5.
Goode CJ, Lovett MK, Haves JE, et al. Use of research-based knowledge in Goode CJ, Lovett MK, Hayes JE, et al. Use of
clinical practice. J Nurs Adm 1987;17:11-18.

clinical practice. J Nurs Adm 1987;17:11-18. Royle JA, Blythe J, Potvin C, et al. Litera
workplace. Comput Nurs 1995;13:25-31.

9 Mitchell A, Janzen K, Pask E, et al. Assessment of nursing research utilization needs in Ontario health agencies. Can J Nurs Adm 1995;8:77-91.

10 Royle JA, Blythe J, DiCenso A, et al. Do nurses have the information resources and skills for research utilization? Can J Nurs Adm 1997;10:9-30.

11 Blythe J, Royle JA. Assessing nurses' information needs in the work environment. Bull Med Libr Assoc 1993;81:433-5.

12 Stephens LC, Selig CL, Jones LC, et al. Research application: teaching staff nurses to use library search strategies. J Contin Educ Nurs 1992:23:24-8.

13 Royle JA, Blythe J, Boblin-Cummings S, et al. Nursing for the twenty-first century: using information technology to enhance nursing practice. Hamilton,
Ontario: McMaster University, 1997 .

14 Dobbins M, Ciliska D, DiCenso A. Dissemination and use of research evidence for policy and practice: a framework for developing, implementing, and evaluating strategies. A report prepared for the Dissemination and Utilization Model Advisory Committee of the Canadian Nurses Association and Health Canada, 1998.

15 Thomson MA. Closing the gap between nursing research and practice [implementation forum]. Evidence-Based Nursing 1998 Jan; $1: 7-8$.

6 Funk SG, Champagne MT, Wiese R, et al. BARRIERS: the barriers to utilization scale. Appl Nurs Res 1991;4:39-45.

17 Funk SG, Tornquist EM, Champagne MT. Barriers and facilitators of research utilization. An integrative review. Nurs Clin North Am 1995;30: 395-407.

18 Bostrom J, Suter WN. Research utilization: making the link to practice. $J$ Nurs Staff Dev 1993;9:28-34.

19 Logan J, Davies B. The staff nurse as research facilitator. Can J Nurs Adm 1995;8:92-110.

20 MacGuire JM. Putting nursing research findings into practice: research utilization as an aspect of the management of change. $J$ Adv Nurs

21 Titler MG, Kleiber C, Steelman V, et al. Infusing research into practice to promote quality care. Nurs Res 1994;43:307-13.

Erratum: In the April 1998 issue of Evidence-Based Nursing, the 2 abstracts related to early hospital discharge (Edmonson et al and Liu $e t a l)$ appeared in the treatment section, but they should have appeared in the causation section. 